\title{
BMJ Open Conceptual framework for personal recovery in mental health among children and adolescents: a systematic review and narrative synthesis protocol
}

\author{
Anna Ballesteros-Urpi, ${ }^{1,2}$ Mike Slade, ${ }^{3}$ David Manley, ${ }^{4}$ Hector Pardo-Hernandez ${ }^{\odot, 6}$
}

To cite: Ballesteros-Urpi A, Slade M, Manley D, et al. Conceptual framework for personal recovery in mental health among children and adolescents: a systematic review and narrative synthesis protocol. BMJ Open 2019;9:e029300. doi:10.1136/ bmjopen-2019-029300

- Prepublication history for this paper is available online. To view these files, please visit the journal online (http://dx.doi org/10.1136/bmjopen-2019029300).

Received 21 January 2019 Revised 14 July 2019 Accepted 17 July 2019
Check for updates

(C) Author(s) (or their employer(s)) 2019. Re-use permitted under CC BY-NC. No commercial re-use. See rights and permissions. Published by BMJ.

For numbered affiliations see end of article.

Correspondence to Dr Hector Pardo-Hernandez; hpardo@santpau.cat

\section{ABSTRACT}

Introduction Personal recovery has been defined as 'a profound personal and unique process for the individual to change their attitudes, values, feelings, goals, abilities and roles in order to achieve a satisfactory, hopeful and productive way of life, with the possible limitations of the illness'. However, research on personal recovery has focused almost exclusively on adults. This project aims to systematically review the available literature on definitions of personal recovery among children and adolescents with mental health conditions and to undertake a narrative synthesis to develop a conceptual framework of recovery.

Methods and analysis Systematic review and narrative synthesis consisting (1) searching scientific literature databases, (2) handsearching, (3) citation tracking, (4) grey literature searching, (5) web-based searching and expert consultation. We will include qualitative and quantitative studies or systematic reviews providing a definition, theoretical or conceptual framework, domains or dimensions of personal recovery among eligible participants. The study will follow standard systematic review methodology for study selection and data extraction. We will assess quality of the evidence using tools appropriate for each study design. We will develop a new conceptual framework using a modified narrative synthesis approach, as follows: (1) describing eligible studies and conducting a preliminary synthesis, (2) determining relationships within and between studies and (3) determining the robustness of the synthesis. Ethics and dissemination We obtained a waiver of approval from our local Research Ethics Committee. Results will be disseminated via publications in international peer-reviewed journals and conference proceedings.

This study will result in a theoretical framework that is based on an exhaustive review of the literature and the input of experts in the field of recovery. We expect that this framework will foster a better understanding of the stages and processes of recovery in children and adolescents with mental health conditions.

PROSPERO registration number CRD42018064087.

\section{INTRODUCTION}

Personal recovery has been defined as 'a profound personal and unique process...
Strengths and limitations of this study

- This systematic review will include both published and unpublished literature, hence reducing the risk of publication bias.

- Duplicate and independent screening and data extraction will minimise the risk of error when identifying eligible studies and extracting relevant data.

- We expect heterogeneity in the included studies which may make it difficult to summarise data for the development of a recovery conceptual framework for children and adolescents with mental health conditions.

to change their attitudes, values, feelings, goals, abilities and roles in order to achieve a satisfactory, hopeful and productive way of life, with the possible limitations of your illness'. ${ }^{1}$ Personal recovery differs from clinical recovery which mainly focuses on reducing symptoms and improving functioning levels. ${ }^{23}$ Although clinical improvement has an impact on personal recovery, healthcare staff can go further by working collaboratively to support mental health service users to live a satisfactory, hopeful and productive life.

In recent years, national health programme, especially in English-speaking countries, have explored the potential that the personal recovery process can offer to increase patient satisfaction and experience. ${ }^{4}$ One of the most accepted theoretical frameworks at present to understand personal recovery is CHIME, a compilation of five interrelated recovery processes Connectedness, Hope and optimism about the future, Identity, Meaning in life and Empowerment $^{5-7}$ (figure 1). CHIME was developed through a systematic review and narrative synthesis of the existing literature on personal recovery frameworks and definitions. ${ }^{5}$ The scientific search of this review 


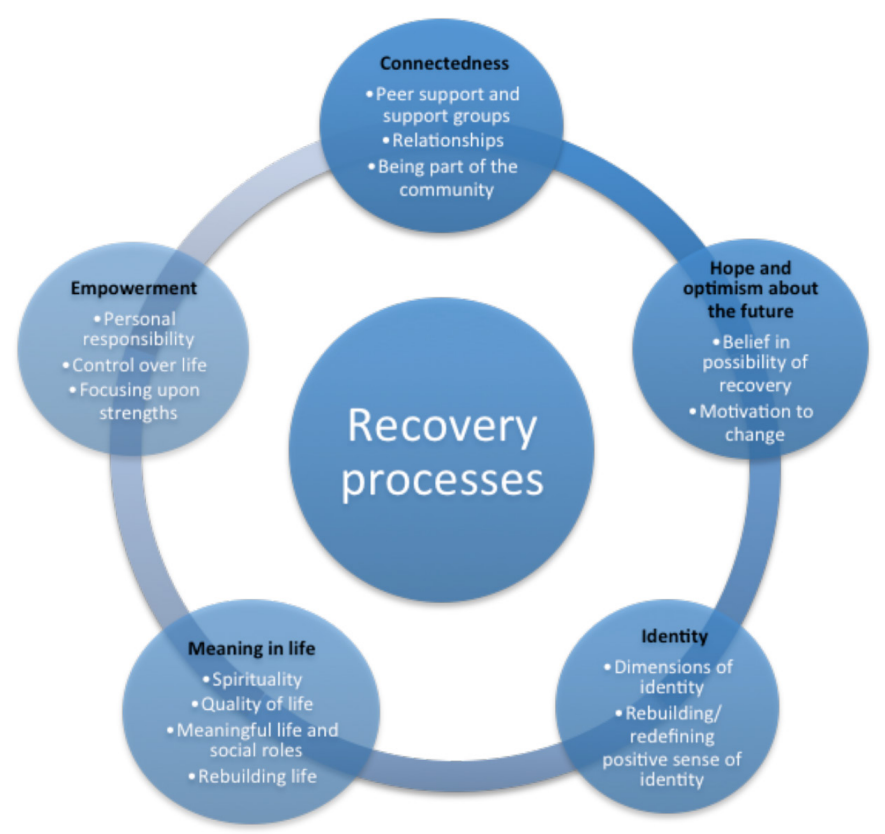

Figure 1 Connectedness, Hope and optimism about the future, Identity, Meaning in life and Empowerment recovery framework.

spanned until 2009, and no studies focused on children or adolescents were identified.

Applying currently available recovery-focused interventions in younger populations may present unique challenges. For instance, connectedness in a child or adolescent is linked to the relationship with their parents and family and to their dependence on many aspects of everyday life. ${ }^{8}$ Similarly, the relationship between children and adolescents with the peer group and the effect of this relationship on the development of identity and meaning in life is not exhaustively reflected in the current recovery approach. ${ }^{9}$ Peers may exert an important influence via a sense of belonging, constructive or destructive relationships with members of the same or opposite sex, bullying and so on. ${ }^{8}$ Regarding identity and meaning in life, childhood and adolescence are stages of change, definition and clarification, whereas for adults identity and definition of self, while ever evolving, are largely set. A diagnosis of a mental illness may create a schism in this developmental process; the process of accepting the condition and dealing with symptoms, the associated stigma and the response of family and peers will influence identity and the definition of self. Recovery, therefore, must recognise this dynamism and reflect progress in this process; not simply a return to the initial state, prior to the diagnosis. ${ }^{8}$

To our knowledge, the only available evidence on recovery frameworks for younger population is a scoping review that aimed to determine whether personal recovery-oriented mental health practice is applicable to children and adolescents with mental health issues. ${ }^{10}$ Authors found that recovery for young people appears to be consistent with CHIME recovery

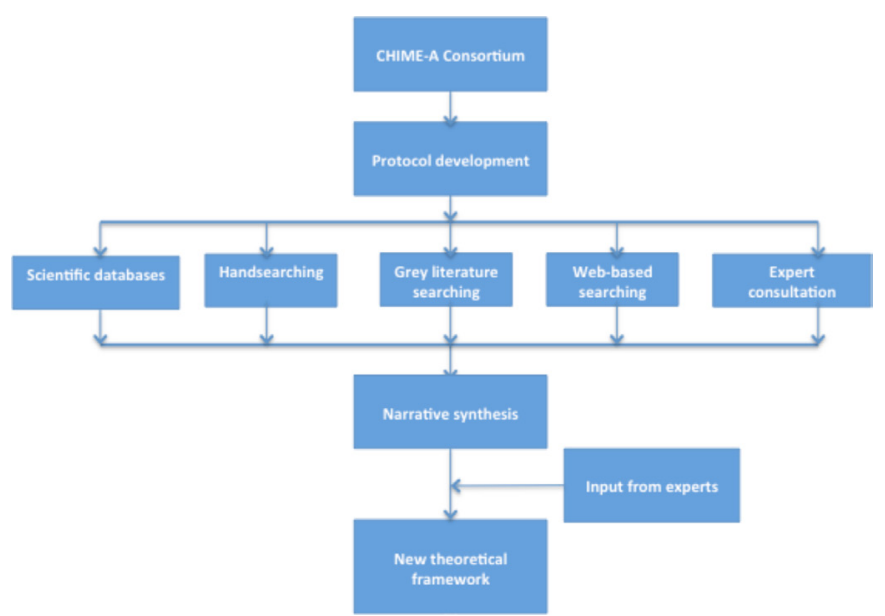

Figure 2 Summary of the systematic review methodology.

framework components, although they highlighted the importance of including parents in the recovery process. This scoping review, however, has methodological limitations, including a very simple search strategy of the available literature (with the terms 'mental health', 'recovery' and either 'child', 'adolescent' or 'CAMHS') and the exclusion of recovery literature focused on specific mental health disorders (eg, psychosis). The study relied heavily on the CHIME recovery framework and did not explore the potential of developing new frameworks.

This project aims to advance the available evidence on recovery among children and adolescents with mental health conditions by systematically reviewing the available literature on the definitions of personal recovery among children and adolescents with mental health conditions 18 years of age or younger. We aim to implement an exhaustive search strategy and to implement a narrative synthesis of this evidence to develop a conceptual framework of recovery specific to this population.

\section{METHODS AND ANALYSIS \\ Study design}

This study is a systematic review with a modified narrative synthesis (figure 2). This review protocol was prepared following the Preferred Reporting Items for Systematic Reviews and Meta-Analyses Protocols (PRISMA-P) ${ }^{11}$ This systematic review will be carried out with the support of CHIME for Adolescents (CHIME-A), an international consortium of professionals with a common interest in the field of mental health recovery among children and adolescents (https://www. researchintorecovery.com/chime-a).

The systematic review will be reported in accordance with the PRISMA statement. ${ }^{12}$

\section{Types of studies}

We will include qualitative or quantitative empirical studies involving at least three participants that provides a definition of personal recovery among people 
experiencing mental health conditions who are 18 or younger; or that provides a theory, theoretical framework, conceptual framework, domains or dimensions of personal recovery. Systematic reviews with similar scopes will also be included. Clinical practice guidelines and editorials will be excluded, as well as studies with one or two participants, since studies with such a small sample size may not be generalisable or achieve saturation. Language of publication will be limited to English and Spanish.

\section{Type of participants}

Participants and health conditions

Individuals $0-18$ years of age who have experienced mental health disorders, either diagnosed or self-reported. We will classify mental health conditions in accordance with the 2017/2018 International Classification of Diseases (ICD)-10, section 'F01-F99 Mental, Behavioural and Neurodevelopmental disorders' which defines mental health disorders as 'a clinically recognisable set of symptoms or behaviour associated in most cases with distress and with interference with personal functions.' (https://www.who.int/classifications/icd/ en/bluebook.pdf?ua=1).

\section{Settings}

Individuals in any setting including but not limited to hospitals, primary care centres or community mental health settings.

\section{Type of outcomes measures}

We will consider the following outcome measures: (1) any definition of personal recovery among participants; (2) a theory, theoretical framework, conceptual framework, domains; (3) dimensions of personal recovery among eligible participants.

\section{Searches}

\section{Scientific databases}

We will perform systematic searches in the most important literature databases as well as those most relevant to the field of mental health and social studies (box 1). All searches will be limited to 2009 onwards to

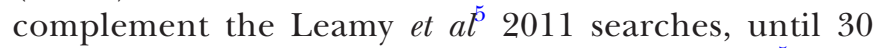
September 2018. We will re-cover the Leamy et a $\tilde{e}^{2} 2011$ searches to verify that no papers on recovery applicable to our population of interest are missed.

The search strategy in its PubMed form is presented in box 2; it will be adapted for the different databases we will consult. This strategy, developed in consultation with a health sciences librarian, was adapted from the CHIME systematic review.

\section{Handsearching}

We will handsearch the table of contents of journals that, based on the experience of the research team, have a record of publishing material on personal recovery (box 1). In addition, we will consult journals publishing a high proportion of studies found to be eligible via

\section{Box 1 Published literature searches}

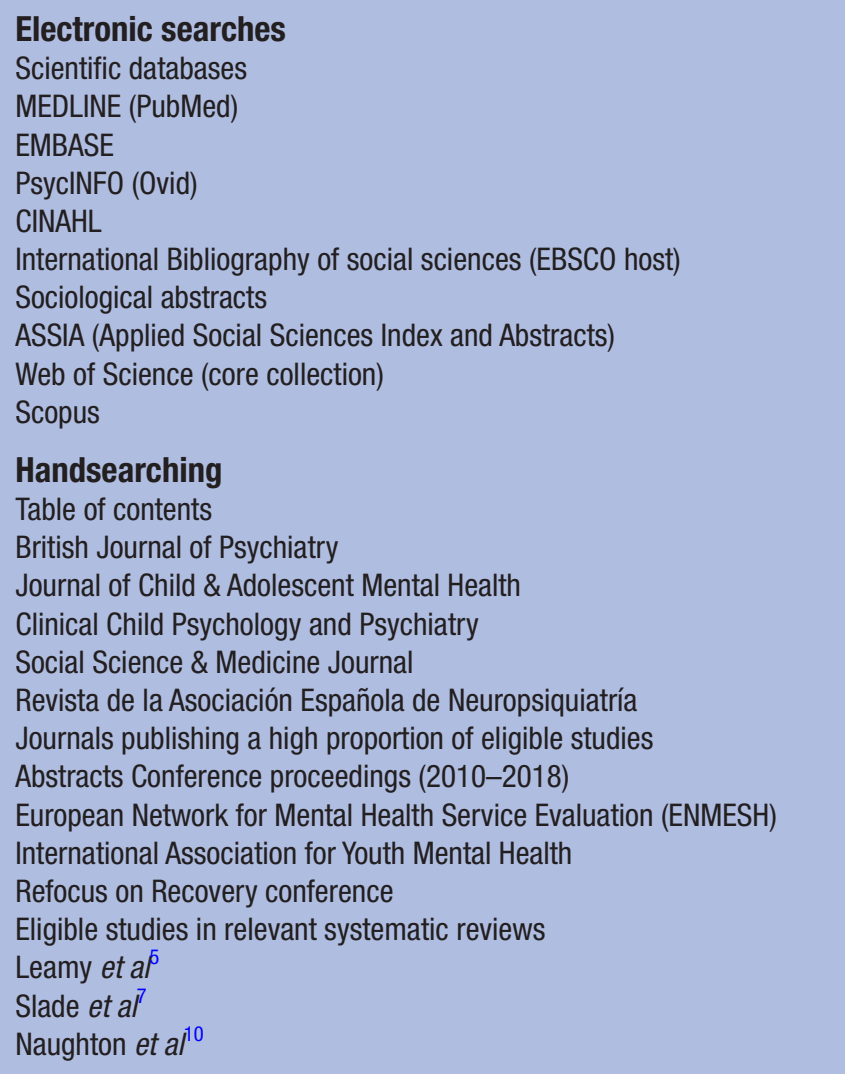

the screening process, as well as included studies in eligible systematic reviews. We will search proceedings from relevant conferences in the field of recovery and mental Health among youth: European Network for Mental Health Service Evaluation, Refocus on Recovery conference and the International Conference on Youth Mental Health. All handsearching will be limited to 2009-2018 (box 1).

\section{Citation tracking}

We will assess all studies citing the Leamy et a $a \tilde{l}$ study, searched via Google Scholar (available at: https:// scholar.google.co.uk/scholar? oi=bibs\&hl=en\&cites= $150376588524122793)$.

We will also screen all studies included in the Leamy et $a \tilde{\varphi}$ review (box 3 ), in the recently published review on this matter ${ }^{10}$ and in another one developed by our research team ${ }^{7}$ (box 2).

\section{Grey literature searching}

We will search OpenGrey ('recovery' and 'mental health' OR 'CHIME'). We will also conduct different Google searches; since we expect a large number of results, we will consider only the first 100 hits (box 3).

\section{Web-based searching}

We will search on-line repositories ('recovery' and 'mental health' OR 'CHIME') with a specific focus on recovery in mental health (box 3). 


\section{Box 2 Search strategy (PubMed version)}

1. Mental health $O R$ mental illness ${ }^{\star} O R$ mental disorder ${ }^{*}$ OR mental disease ${ }^{*} \mathrm{OR}$ mental problem

2. Recover*

3. theor ${ }^{*} \mathrm{OR}$ framework ${ }^{\star} \mathrm{OR}$ model $\mathrm{OR}$ dimension $\mathrm{OR}$ paradigm $\mathrm{OR}$ concept $^{\star}$

4. (\#2 AND \#3)

5. (\#1 AND \#4)

6. Psychol* health $\mathrm{OR}$ psychol ${ }^{\star}$ illness* $\mathrm{OR}$ psychol ${ }^{*}$ disorder $\mathrm{OR}$ psychol* problem OR psychiatr* health, OR psychiatry* illness* OR Psychiatr* disorder OR psychiatr* problem

7. theme* $O R$ stages $O R$ processes

8. (\#2 AND \#7)

9. (\#6 AND \#8)

10. CHIME [(tiab])

11. Minors $O R$ minors* $O R$ boy $O R$ boys $O R$ boyfriend $O R$ boyhood $O R$ girl* $O R$ kid OR kids $O R$ child $O R$ child* OR children* OR schoolchild* OR schoolchild OR school child[(tiab]) OR school child*[(tiab]) OR adolescen* $O R$ juvenil ${ }^{*} O R$ youth* $O R$ teen ${ }^{*} O R$ under*age ${ }^{\star} O R$ pubescen $^{*} \mathrm{OR}$ pediatricspaediatrics[(mh]) OR pediatricpaediatric* OR paediatric* OR peadiatric* OR school [(tiab]) OR school*[(tiab]) OR prematur* OR preterm*

12. (\#5 OR \#9 OR \#10) AND (\#11)

\section{Expert consultation}

We will consult experts in the field as identified by the CHIME-A consortium about potentially eligible studies (box 3).

\section{Data collection and analysis}

Study selection

We will use EndNote to collate all references and remove duplicates. Two independent reviewers will assess all titles and abstracts to determine whether a full text review is needed. We will solve discrepancies by consensus or

\section{Box 3 Other sources that will be searched}

\section{Citation tracking}

Studies citing Leamy et a ${ }^{\bar{p}}$

Grey literature ("recovery" and "mental health" OR "CHIME")

OpenGrey

Google searches ("recovery" and "mental health" OR "CHIME") ("recovery" and "mental health" and "child") ("recovery" and "mental health" and "adolescent") 100 hits from a Google search

\section{Web-based searching}

Scottish Recovery Network

Recovery Devon

Boston University Repository of Recovery Resources

Rethink

National Mental Health Development Unit

Social Perspectives Network

\section{Expert consultation}

Consult experts in the field consulting a third investigator. Two independent reviewers will then review all papers eligible for full-text assessment. Discrepancies will be solved by consensus or by consulting a third investigator.

\section{Data extraction and management}

For each study, we will extract its full reference, description of participants (including country and age profile), sample size, methodology, service setting and definition of recovery. Data extraction will be performed individually with an independent author verifying a random $10 \%$ sample to ensure accuracy. If we identify any missing data, we will contact study authors.

\section{Analysis of subgroups}

We will analyse subgroups per age, specifically participants 12-18 years of age. We will also assess studies including patients with ADHD or autism separately. In both cases we will attempt to determine if there are any differences in study findings.

\section{Risk of bias assessment}

Due to the expected heterogeneity of potentially eligible studies, we will adopt a multi-pronged approach to assess risk of bias. We will use the Critical Appraisal Skills Programme (CASP) checklist for qualitative studies; ${ }^{13}$ we will rate studies presenting serious concerns in over two CASP items as having 'serious methodological limitations' and those presenting serious concerns in two items as having 'moderate methodological limitations'. The rest will be classified as high quality. We will use the Confidence in the Evidence from Reviews of Qualitative Research (CERQual) tool to assess the overall quality of the evidence of qualitative studies. For quantitative and mixed methods studies, we will use the Mixed Methods Appraisal Tool. ${ }^{14}$ We will not assess the quality of doctoral theses, books, book chapters and government reports. We will use risk of bias assessment results to gauge the confidence we can place on the identified evidence, research gaps and guidance for interpreting findings. Additionally, we will analyse separately studies classified as of high quality.

\section{Data synthesis and analysis}

We will undertake analysis using narrative synthesis, ${ }^{15}$ following the approach and building on the theory underpinning the CHIME Framework. First, we will comprehensively assess and summarise the results of eligible studies. We will then conduct a preliminary thematic synthesis of the definitions, theories, theoretical frameworks, conceptual frameworks, domains or dimensions identified in the eligible studies. We will do so by grouping studies according to study type and setting; we will tabulate results and identify conceptual overlaps, similarities and differences and will explore possible explanations for these, considering narratives at the level of form, structure and content. We will identify overarching themes and related subthemes and will determine the frequency with which themes appear in the included papers via vote counting. The preliminary synthesis will then be refined and discussed among study 
authors, with a specific emphasis on common themes and related sub-themes, resulting in an initial conceptual framework of recovery. To minimise bias in the analysis process, the primary analyst team have multi-professional (social worker, nurse, psychologist) and multidisciplinary (methodologist, educational, health services research, management) expertise, and the wider analyst team also involves occupational therapy, psychometric, peer research, sociology and health research expertise. The analyst team include researchers involved in the development of the CHIME Framework and researchers with no previous involvement. Afterwards, we will explore relationships within and between studies in relation to this initial conceptual framework, which will allow further discussion and the development of an overarching framework. We will explore whether variability in study design, population or setting can explain differences within studies and will contrast emerging themes against the preliminary synthesis in order to identify discrepancies and to assess robustness. Lastly, we will assess the robustness of the overarching framework through consultation with experts and study authors and by comparing results in higher-quality studies with the overall synthesis.

\section{Patient and public involvement}

Patients were not involved in the development of this protocol. However, the research question we address in this project aims to fill an evidence gap on the concept of recovery among children and adolescents. We expect that the definition of recovery we aim to develop will better inform services to youth living with mental health conditions.

\section{Ethics and dissemination}

This protocol was written following the PRISMA-P guidelines $^{11}$ the review will be reported according to the PRISMA statement. ${ }^{12}$ We plan to disseminate the findings of this systematic review through peer-reviewed journal publications and conference presentations.

This systematic review will result in a new theoretical framework that is based on an exhaustive review of the available literature and on the input of international experts in the field of recovery among children and adolescents. We expect that the new framework will foster a better understanding of the stages and processes of recovery in this population. In addition, the conceptual framework can contribute to the development of measures of personal recovery among children and adolescents. The conceptual framework can provide a foundation for developing standardised recovery measures, and can serve as the basis for a future measure to evaluate the contribution of mental health services to mental health recovery among children and adolescents.

\section{Author affiliations}

${ }^{1}$ Department of Child and Adolescent Psychiatry and Psychology, Institute of Neurosciences, Hospital Clínic de Barcelona, Barcelona, Spain

${ }^{2}$ Department of Education, Universitat de Barcelona, Barcelona, Spain

${ }^{3}$ School of Health Sciences, Institute of Mental Health, University of Nottingham, Nottingham, UK
${ }^{4}$ Nottinghamshire Healthcare NHS Foundation Trust, Nottingham, UK

${ }^{5}$ Iberoamerican Cochrane Centre, Sant Pau Biomedical Research Institute (IIB Sant Pau), Barcelona, Spain

${ }^{6}$ CIBER of Epidemiology and Public Health (CIBERESP), Barcelona, Spain

Acknowledgements Anna Ballesteros Urpi is a PhD candidate in the Department of Education at the Universitat de Barcelona (Barcelona, Spain). Mike Slade is supported by the National Institute for Health Research Nottingham Biomedical Research Centre. Authors would like to thank the members of the CHIME-A Consortium for their input during the development of this manuscript.

Contributors Study idea: ABU, MS, DM, HPH. Study design: ABU, MS, DM, HPH. Drafting of the first version of the article: $A B U$. Critical revision of the article: $A B U$, MS, DM, HPH. Final approval of the article: ABU, MS, DM, HPH.

Funding Anna Ballesteros Urpi is supported by a Sabbatical Leave Grant from Hospital Clinic (Barcelona, Spain).

Competing interests None declared.

Patient consent for publication Not required.

Ethics approval We have obtained a waiver of approval from the Clinical Research Ethics Committee at the Hospital Clínic of Barcelona (Barcelona, Spain).

Provenance and peer review Not commissioned; externally peer reviewed.

Open access This is an open access article distributed in accordance with the Creative Commons Attribution Non Commercial (CC BY-NC 4.0) license, which permits others to distribute, remix, adapt, build upon this work non-commercially, and license their derivative works on different terms, provided the original work is properly cited, appropriate credit is given, any changes made indicated, and the use is non-commercial. See: http://creativecommons.org/licenses/by-nc/4.0/.

\section{REFERENCES}

1. Anthony WA. Recovery from mental illness: the guiding vision of the mental health service system in the 1990s. Psychosocial Rehabilitation Journal 1993;16:11-23.

2. Slade M, Bird V, Le Boutillier C, et al. REFOCUS trial: protocol for a cluster randomised controlled trial of a pro-recovery intervention within community based mental health teams. BMC Psychiatry 2011;11:1-13.

3. Turton P, Demetriou A, Boland W, et al. One size fits all: or horses for courses? Recovery-based care in specialist mental health services. Soc Psychiatry Psychiatr Epidemiol 2011;46:127-36.

4. HM Government. New horizons. A shared vision for mental health 2009.

5. Leamy M, Bird V, Le Boutillier C, et al. Conceptual framework for personal recovery in mental health: systematic review and narrative synthesis. Br J Psychiatry 2011;199:445-52.

6. Bird V, Leamy M, Tew J, et al. Fit for purpose? validation of a conceptual framework for personal recovery with current mental health consumers. Aust N Z J Psychiatry 2014;48:644-53.

7. Slade M, Leamy M, Bacon F, et al. International differences in understanding recovery: systematic review. Epidemiol Psychiatr Sci 2012;21:353-64.

8. Ward D. 'Recovery': does it fit for adolescent mental health? J Child Adolesc Ment Health 2014;26:83-90.

9. Tew J, Ramon S, Slade M, et al. Social factors and recovery from mental health difficulties: a review of the evidence. Br J Soc Work 2012;42:443-60.

10. Naughton JNL, Maybery D, Sutton K. Review of child and adolescent mental health recovery literature: concordance and Contention. $J$ Psychosoc Rehabil Ment Health 2018;5:151-8.

11. Moher D, Shamseer L, Clarke M, et al. Preferred reporting items for systematic review and meta-analysis protocols (PRISMA-P) 2015 statement. Syst Rev 2015;4:1.

12. Moher D, Liberati A, Tetzlaff J, et al. Preferred reporting items for systematic reviews and meta-analyses: the PRISMA statement. BMJ 2009;339:b2535.

13. Spittlehouse C, Acton M, Enock K. Introducing critical appraisal skills training in UK social services: another link between health and social care? J Interprof Care 2000;14:397-404.

14. Pluye P, Robert E, Cargo M. Proposal: a mixed methods appraisal tool for systematic mixed studies reviews. Montréal: McGill University, 2011: 2. 1-8.

15. Popay J, Roberts H, Sowden A. Guidance on the conduct of narrative synthesis in systematic reviews. A product from the ESRC methods programme version; 2006. 